\title{
El rol de la reflexión en la práctica pedagógica: percepciones de docentes de idiomas en formación
}

\author{
CLARA INÉS GONZÁLEZ MARÍN" \\ NATHALIA MARÍN PALACIO \\ MIGUEL ANTONIO CARO GUTIÉRREZ ${ }^{* * *}$
}

Recepción: 29 de noviembre de 2017

Aprobación: 30 de abril de 2018

Forma de citar este artículo: González, C.I., Marín, N. \& Caro, M. A. (2018). El rol de la reflexión en la práctica pedagógica: percepciones de docentes de idiomas en formación. Cuadernos de Lingüística Hispánica, (32), 217- 235.

* Artículo de investigación.

** Administradora de Negocios de la Universidad Autónoma de Manizales, licenciada en Educación Básica con énfasis en Lengua Extranjera de la Universidad El Bosque de Bogotá y magíster en Lingüística de la Universidad Tecnológica de Pereira. Actualmente labora como profesora de tiempo completo en el programa Licenciatura en Bilingüismo con Énfasis en Inglés de la Universidad Tecnológica de Pereira. Correo electrónico: claraigoma@utp.edu.co

*** Licenciada en Lengua Inglesa de la Universidad Tecnológica de Pereira. Actualmente trabaja en el Instituto de Lenguas Extranjeras (ILEX) de la Universidad Tecnológica de Pereira. Correo electrónico: namarin@utp.edu.co.

**** Licenciado en Lengua Inglesa de la Universidad Tecnológica de Pereira. Ha trabajado en diferentes colegios oficiales y privados de la ciudad de Pereira como Escuela Normal Superior, INEM Felipe Pérez y La Salle, y en institutos de lenguas como el Centro Colombo Americano, actualmente labora en el instituto de lenguas American School Way sede Pereira. Correo electrónico: macaro@utp.edu.co. 


\section{Resumen}

El presente estudio de caso tuvo como propósito conocer las percepciones de los estudiantes de un programa de enseñanza de inglés frente al rol de la reflexión en sus prácticas educativas e indagar sobre la estructura de la reflexión, incluyendo los aspectos que los practicantes más comúnmente comprenden a la hora de reflexionar. Para este propósito, fueron seleccionados nueve estudiantes practicantes, a los que se les analizaron ensayos reflexivos, reflexiones realizadas después de clase y la participación en un cuestionario virtual. Los resultados demostraron que los estudiantes practicantes percibieron la reflexión como un elemento fundamental e indispensable para encontrar soluciones frente a aspectos negativos de sus clases. Si bien los practicantes señalaron como una de sus motivaciones para reflexionar el hecho de ser un ejercicio obligatorio en la asignatura, también reconocieron la importancia de la misma como un elemento fundamental para su desarrollo profesional docente. El esquema más común seguido por los estudiantes en sus reflexiones fue la descripción de eventos de clase como paso previo a la reflexión. Como conclusión, se pudo establecer que los estudiantes aún se encuentran en un nivel básico de reflexión, lo que impulsó al programa de práctica a trabajar más sobre este componente.

Palabras clave: reflexión, practicantes, desarrollo profesional, enseñanza.

\section{The Role of Reflection in Pedagogical Practice: Perceptions of Language Teachers in Training}

\section{Abstract}

The purpose of this case study was to understand the perceptions of students of an English teaching program in the face of the role of reflection in their educational practices and to investigate the structure of reflection, including the aspects that practitioners most commonly understood when they reflected. For this purpose, nine practicing students were selected, who were analyzed reflective essays, reflections made after class and participation in a virtual questionnaire. The results showed that the practicing students perceived the reflection as a fundamental and indispensable element to find solutions to negative aspects of their classes. Although the practitioners pointed out as one of their motivations to reflect the fact of being a mandatory exercise in the subject, they also recognized the importance of it as an overriding element for their professional teaching development. The most common scheme followed by students in their reflections was the description of class events as a preliminary step to reflection. In conclusion, it was established that the students are still at a basic level of reflection, which prompted the practice program to work more on this component.

Keywords: reflection, practitioners, professional development, teaching. 


\section{Le rôle de la réflexion dans la pratique pédagogique : perceptions des enseignants de langues, en formation}

\section{Résumé}

Cette étude de cas a eu comme but, le fait de connaitre les perceptions des étudiants d'un programme d'enseignement d'anglais, face au rôle de la réflexion dans leurs pratiques éducatives, et enquêter sur la structure de la réflexion, en incluant les aspects que les stagiaires comprennent le plus souvent au moment de réfléchir. . À cette fin, on a choisi neuf stagiaires, dont on a analysé des essais réflexifs. Ces réflexions-ci avaient été faites après le cours et la participation à un questionnaire virtuel. Les résultats ont montré que les stagiaires ont perçu la réflexion comme un élément fondamental et indispensable pour trouver des solutions face aux aspects négatifs de leurs cours. Bien que les stagiaires aient signalé que le fait d'être un exercice obligatoire des cours, était une des motivations pour réfléchir, ils ont aussi reconnu l'importance de la réflexion comme un élément fondamental pour leur développement professionnel pour devenir enseignants. Le schéma le plus commun pour les étudiants dans leurs réflexions, a été la description d'évènements de cours, comme un pas avant la réflexion. Comme conclusion, on a pu établir que les étudiants sont encore à un niveau de base de réflexion, ce qui a stimulé le programme de pratique à travailler davantage sur ce composant.

Mots clés: réflexion, stagiaires, développement professionnel, enseignement

\section{O papel da reflexão na Prática Pedagógica: Percepções Dos Professores de Línguas no Treinamento}

\section{Resumo}

0 objetivo deste estudo de caso foi compreender as percepções de estudantes de um programa de ensino de inglês em relação ao papel da reflexão em suas práticas educacionais e investigar a estrutura de reflexão, incluindo os aspectos que os profissionais mais comumente entendem a 0 tempo para refletir. Para tanto, foram selecionados nove alunos praticantes, e foram analisados seus ensaios reflexivos, reflexões realizadas após a aula e participação em um questionário virtual. Os resultados mostraram que os estudantes praticantes perceberam a reflexão como um elemento fundamental e indispensável para encontrar soluções para aspectos negativos de suas aulas. Embora os profissionais apontassem como uma de suas motivações para refletir o fato de ser um exercício obrigatório no tema, eles também reconheceram a importância do mesmo como elemento fundamental para o desenvolvimento do ensino profissional. 0 esquema mais comum seguido pelos estudantes em suas reflexões foi a descrição dos eventos de classe como um passo preliminar para a reflexão. Em conclusão, foi estabelecido que os alunos ainda estão em um nível básico de reflexão, o que levou o programa de prática a trabalhar mais neste componente.

Palavras-chave: reflexão, profissionais, desenvolvimento profissional, ensino. 


\section{Introducción}

En la actualidad, el concepto de práctica reflexiva es cada vez más popular en los programas de desarrollo profesional docente. El Ministerio de Educación Nacional establece en la resolución 02041 de 2016 las características de calidad de los programas de licenciaturas del país, señalando que la institución de educación superior debe contar con una organización que favorezca la formación y retroalimentación de calidad de los futuros docentes, donde la práctica pedagógica se organice de tal manera que en este proceso el estudiante "sea protagonista de una reflexión sistemática sobre su propia práctica para mejorarla y organizar su aprendizaje" (p.7).

En este sentido, distintos autores (p. ej. Madrid, 2004; Páez, 2001) han destacado las cualidades y destrezas que un docente de idiomas debe adquirir durante su proceso de formación, como la competencia pedagógica, comunicativa (Páez, 2001) el saber ser, hacer y vivir (Delors, 1999), la relación entre teoría y práctica (Madrid, 2004), entre otras, que permiten la idealización de un docente de idiomas de alta calidad. Sin embargo, el rol de la reflexión como un principio para el mejoramiento de la práctica docente y el desarrollo profesional no suele ser muy mencionado, a pesar de que este promete ser trascendental en el proceso de formación docente, no solo por su papel en el reconocimiento y aprendizaje sobre los errores metodológicos ocurridos en la enseñanza, sino también porque involucra el progreso y las dificultades en el aprendizaje de los alumnos, lo que puede conllevar distintas soluciones.

En efecto, solo a través de este recurso es posible desarrollar el pensamiento crítico y la solución de problemas, así como identificar las disparidades entre teoría y práctica que pueden conducir a la adaptación de metodologías ya existentes al contexto presente. Además, el hecho de reflexionar sobre el acto pedagógico tiende también a descubrir posibles frustraciones y actitudes del practicante que a menudo no suelen ser analizadas con detenimiento y pueden ser las causantes de que la planeación o ejecución de las clases simplemente no funcionen o no resulten como se ha previsto.

Por ello, el propósito de este estudio es evaluar cómo el papel de la reflexión puede impactar la formación y el desarrollo docente en un grupo de estudiantes que aspiran a ser docentes de inglés, durante su práctica pedagógica con estudiantes de primaria, teniendo en cuenta sus actitudes, pensamientos y sentimientos con respecto a esta experiencia, y a su vez, analizar cómo a través de la reflexión, se puede determinar si existen vacíos y brechas entre la teoría (referida a la enseñanza de una lengua extranjera) y la práctica en el contexto de las escuelas públicas en Colombia. Estos objetivos se plantean por medio de la pregunta general de investigación ¿cuál es la percepción de los practicantes acerca de la re- 
flexión como parte de su crecimiento profesional como docentes de inglés? Y las siguientes preguntas específicas de investigación ¿qué conjunto de acciones describen o incorporan los practicantes en sus reflexiones después de clase? y ¿qué apreciación tienen los practicantes sobre la relevancia y contextualización de la teoría vista dentro de las sesiones del programa de licenciatura en inglés durante las prácticas pedagógicas?

\section{Marco teórico}

\subsection{Formación y preparación docente en lenguas extranjeras}

El proceso de profesionalización docente requiere de diferentes aspectos educativos que buscan fortalecer las habilidades personales y epistemológicas del educador, como lo afirma Peters (1966), quien define la educación docente como "un proceso de aprendizaje que desarrolla aspectos: morales, culturales, sociales e intelectuales de la persona que es tomada como un individuo y miembro de la sociedad" (p. 3). Sin embargo, es importante resaltar que el término "educación docente" ha sido tomado con una relación semántica igualitaria a la de "formación docente"; puesto que el último término, según Ur (1999), se refiere al sobreénfasis de técnicas y habilidades, mientras que el profesor profesional necesita desarrollar y aplicar teorías, estar consciente de las opciones teóricas del momento y poseer habilidad de tomar decisiones. Estas destrezas son fundamentales y deben estar claras para poder ser usadas durante sus prácticas pedagógicas.

\subsection{El rol de la práctica en profesores noveles}

Durante el proceso de formación de un licenciado en lenguas, algunas teorías y herramientas son proporcionadas desde la academia para ser aplicadas a la hora de salir al campo laboral; no obstante, ningún profesor novel suele estar completamente preparado para asumir el rol de docente hasta que es expuesto a una práctica pedagógica; siendo esta un lugar en el que el practicante se enfrenta a un contexto real (Zeichner, 1996; citado en Fekede \& Gemechis, 2009) y que incorpora un periodo de inquietud y desarrollo crítico en el cual el profesor novato decide si la enseñanza es la carrera de su vida (Faez \& Valeo, 2012). Del mismo modo, Pitton (2006) afirma que "el éxito de un nuevo profesor está críticamente ligado a sus primeras experiencias de enseñanza" (p. 2). De acuerdo con lo anterior, es posible deducir que durante la práctica pedagógica se obtienen las primeras experiencias de enseñanza y en ella se define la decisión y el rumbo del profesor principiante.

En medio del desarrollo de la práctica, este profesor puede afrontar múltiples experiencias positivas y negativas que contribuirán a su desarrollo como profesional. No obstante, el practicante puede enfrentarse en ocasiones a una realidad diferente a la imaginada y es en donde, según Veenman (1998), este sufre el llamado "shock de la realidad", 
entendido como una reacción negativa inesperada frente a la práctica. Este concepto fue también propuesto por Lortie (1975), quien manifiesta que esta reacción es un fenómeno que no solo se da en el sector educativo, sino también en cualquier profesión, al encontrarse con las nuevas responsabilidades que deberá asumir como profesional. En relación con lo anterior, la reacción denominada "shock de la realidad" es un fenómeno completamente natural que podría enfrentar cualquier practicante en su proceso de adaptación y descubrimiento; sin embargo, a lo largo de su proyecto de vida, entrará a evaluar sus apreciaciones con respecto a las brechas encontradas entre sus perspectivas y el contexto real al que se enfrenta, además de sus reacciones frente a ellas.

En contraste con Veenman (1998) y su concepto de "shock de la realidad", Eyers (2004, citado en Fekede \& Gemechis, 2009) plantea los resultados positivos que se pueden extraer de la práctica en profesores noveles, declarando que las prácticas ayudan al profesor principiante a centrarse en los tres dominios del aprendizaje, "conocimiento de contenido, conocimiento profesional y el conocimiento y las habilidades necesarias para funcionar como profesionales capaces y cuidadores en las escuelas" (p. 111). Como se evidencia en lo mencionado, el rol de la práctica puede afectar positiva o negativamente al profesor novato, permitiéndole experimentar y tomar decisiones, así como adquirir herramientas y conocimientos que le ayuden a su desarrollo profesional; sin embargo, este profesor debe estar atento y siempre dispuesto a mejorar. De este modo, Richards y Lockhart (2007) invitan al profesor novel a reflexionar acerca de su proceso y a encontrar posibles brechas en sus espacios de clase para trabajar en pro de su mejoramiento por medio de la enseñanza reflexiva.

\subsection{Enseñanza reflexiva}

Para comprender el concepto de la enseñanza reflexiva, es necesario empezar por las bases que vinieron como concepción ideológica de John Dewey hacia la acción reflexiva de los maestros (Dewey, 1910; citado por Zeichner \& Liston, 1996), quien sostuvo que este proceso inicia cuando los docentes se enfrentan a una dificultad, algún incidente 0 experiencia que no puede ser resuelta inmediatamente; Munby y Russell (1990) denominaron este fenómeno como puzzles of practice (acertijos de la práctica), en el cual los maestros intentan tomar distancia para analizar sus experiencias, ya que son influenciados por una sensación de incertidumbre e incomodidad. Como veremos, este "tomar distancia" se efectuará durante la acción o después de completarla.

Es necesario que esta acción de autoobservación sea tomada como un hábito, pues según Richards y Lockhart (2007), "cuando la reflexión crítica es vista como un proceso continuo y rutinario en la enseñanza, permite que el profesor se sienta con más confianza 
en probar diferentes opciones y evaluar los efectos de la enseñanza" (p. 4); es decir, que la reflexión constante sobre las acciones hechas en clase brinda seguridad al maestro y permite experimentar y evaluar nuevas didácticas que enriquecerán su quehacer como docente. Por otro lado, Dewey (1933) hizo una distinción importante entre la acción rutinaria y la acción reflexiva, en la cual expresa que la acción-rutina es guiada principalmente por impulso, tradición y autoridad, siendo un ejemplo claro cuando en un colegio se limitan los espacios, las herramientas y las clases de una manera rígida y homogénea, dando por hecho que los profesores seguirán al pie de la letra un protocolo que encapsula el acto de la enseñanza y no se estimula a la reflexión de las estrategias pedagógicas o a las necesidades de los estudiantes. En contraste, Dewey (1933, citado en Zeichner \& Liston, 1996) argumenta que "la acción reflexiva implica la consideración activa, persistente y cuidadosa de cualquier creencia o práctica, tomando en cuenta las razones que la sostienen y las consecuencias que puede tener a futuro" (p. 10).

En otras palabras, el autor asegura que la reflexión tiene en cuenta cualquier arraigo mental y medita sobre los motivos que lo provocan y sus posibles implicaciones para así actuar sobre este. Adicionalmente, Greene (1986) explicó que la enseñanza reflexiva incluye intuición, emoción y pasión, y no es algo que se pueda agrupar sistemáticamente como una serie de técnicas para que el maestro las aplique.

Por otra parte, Richards y Lockhart (2007) sostienen que "si los profesores están activamente envueltos en reflexionar sobre qué está pasando en sus propias clases, ellos estarán en posición de descubrir si hay una brecha o falencia entre lo que enseñan y lo que aprenden sus alumnos" (p. 4). Dichas explicaciones amplifican la percepción de la reflexión, puesto que no solo se limita a enfocarse en qué estuvo mal dentro de la clase, sino que evalúa el proceso de aprendizaje y enseñanza.

Finalmente, el aula de clase debe convertirse en un laboratorio de observación de eventos educativos, ya que la reflexión puede ser vista como un tipo de investigación, tal como lo proponen Cross y Angelo (1988). Para estos autores, la premisa básica de la investigación en los salones de clase es que los profesores usen sus aulas como laboratorios para estudiar el aprendizaje, para así convertirse en observadores hábiles y sistemáticos de cómo los estudiantes aprenden en sus clases.

En conclusión, los docentes deben desarrollar un gran nivel de experticia en cuanto a la observación, ser hábiles e intuitivos, puesto que a través de la identificación de los problemas en el aula se puede llegar a una o varias soluciones con técnicas que tomen en cuenta las variables emocionales que el maestro haya determinado a través de su intuición y pasión. 


\subsection{Niveles de reflexión}

En el quehacer docente se pueden distinguir tres momentos en que se lleva a cabo la reflexión, los cuales fueron clasificados por Schön (1984), Killion y Todnem (1991) como reflexión en la acción, reflexión sobre la acción y reflexión para la acción.

Según Domingo y Gómez (2015), dichos investigadores distinguieron las dos primeras reflexiones como de índole reactiva, y se diferencian entre ellas solo por el tiempo en que se realiza la reflexión, pero ambas obligan al profesor a reaccionar con rapidez 0 inmediatez. En particular, la reflexión en la acción se refiere al proceso de interpretar, analizar y proveer soluciones a los problemas mientras que se está realizando la acción; por otro lado, la reflexión sobre la acción tiene lugar después de que el evento ha ocurrido. A través de esta reflexión se puede encontrar el significado y un sentido a lo que estamos haciendo (Schön, 1987; Ghaye \& Ghaye, 1998). Killion y Todnem (1991), por su parte, definieron el término reflexión para la acción como pensar en futuras acciones con la intención de mejorar o cambiar nuestra práctica docente.

Igualmente, Hatton y Smith (1995) proponen tres niveles de reflexión práctica de acuerdo con la capacidad reflexiva de un profesor, siendo estos la reflexión técnica, la reflexión práctica y la reflexión crítica. La reflexión técnica es definida como el primer nivel y tiene como fin aprovechar las habilidades que se poseen para solucionar un problema. Por medio de la autoobservación, el docente puede encontrar problemas en su quehacer docente y llegar a conclusiones. En cuanto a la reflexión práctica, el profesor va más allá, cuestionando y comparando sus creencias previas con lo que actualmente enfrenta en sus prácticas y se preocupa por conocer las consecuencias que desencadena cada una de sus acciones; por lo cual se hace preguntas como iqué tipo de acción debo emprender frente a esta problemática? ¿Cómo debo hacerlo? y ¿Qué tipo de ayuda necesito de otros? Siendo consciente de que por medio de la reflexión no es posible encontrar siempre soluciones certeras, pero hace que esta sea un vehículo para la acción (Hussein, 2006).

Por último, la reflexión crítica, considerada como el máximo nivel de reflexión, busca que el practicante se involucre autónomamente en la construcción de su comunidad. De esta manera, el profesor en formación se preocupa porque su deber repercuta positivamente en las vidas de sus estudiantes y la comunidad, siendo de su interés conducir al mejoramiento de la calidad de vida de la sociedad por medio del fortalecimiento de valores, haciendo un análisis personal sobre cómo impactarla desde un ámbito social, político o cultural (Hatton \& Smith, 1995). 


\section{Metodología}

\subsection{Tipo de estudio}

El presente estudio tendrá como base el análisis de percepciones de un grupo de estudiantes de Licenciatura en Lengua Inglesa, lo que significa que es una investigación cualitativa de caso, ya que el método cualitativo analiza factores sociales, pensamientos y creencias (Lodico, Spaulding, \& Voegtle, 2006), además considera el contexto y la descripción de acciones y actitudes de los participantes, haciendo inviable la recolección de datos por medio de métodos cuantitativos (Fraenkel, Wallen \& Hyun 2012).

\subsection{Contexto y participantes}

Para este artículo se analizó un grupo de estudiantes de noveno semestre de una Licenciatura en Lengua Inglesa, quienes estaban tomando la asignatura Práctica Pedagógica Dirigida. En esta, los practicantes enseñaban inglés como lengua extranjera en distintas instituciones educativas públicas del sector y tenían como población específica niños de primaria entre los 6 y los 11 años de edad. Estas lecciones eran impartidas dos veces por semana con intensidad de 50 a 60 minutos por lección. Los estudiantes practicantes debían planear la clase, ejecutarla y hacer reflexiones escritas después de terminada la clase, con el fin de incentivar la autocrítica y la reflexión frente a su didáctica. Con este propósito, nueve estudiantes (seis mujeres y tres hombres) fueron seleccionados, y los contenidos de sus reflexiones y ensayos reflexivos fueron analizados; solo siete de ellos participaron en un cuestionario virtual. Estos estudiantes fueron escogidos por medio de un muestreo no aleatorio, señalado por Fraenkel et al. (2012), en el que se seleccionan los participantes según el criterio de los investigadores, y para ello tuvieron en cuenta sus personalidades y el desempeño en otras asignaturas durante su carrera.

\subsection{Recolección de datos}

Con el propósito de recolectar datos para esta investigación, fueron usados los siguientes métodos:

2.3.1 Cuestionarios virtuales: estos fueron realizados con el fin de detectar las percepciones que tenían los practicantes con respecto al rol de la reflexión en sus lecciones y de definir la relación encontrada entre teoría y práctica en el contexto colombiano. Se elaboraron cuestionarios semiestructurados, que fueron pilotados previamente para ayudar a determinar la pertinencia de las preguntas. Estos fueron suministrados por medio de formularios Google. 
2.3.2 Reflexiones escritas: consistieron en una serie de escritos que los practicantes debían hacer luego de cada sesión de clase en el colegio asignado para la práctica; en ellos, los noveles deberían responder a las preguntas ¿Qué salió bien durante la sesión? ¿Qué lo evidencia? ¿Qué aspecto debe mejorarse? y ¿Qué podría hacerse en próximas sesiones? Fue analizado el conjunto de tres reflexiones por persona (es decir, se analizaron en total 27 reflexiones, correspondientes a las clases 5,7 y 9) por medio de una rúbrica que pretendió examinar las percepciones de los practicantes, así como el impacto y la relevancia que tienen estas en el crecimiento profesional de los noveles seleccionados. Las reflexiones se presentaron en inglés y se traducen por los autores en los hallazgos.

2.3.3 Ensayos reflexivos: cada practicante participante presentó dos ensayos reflexivos, uno al inicio y otro al final del semestre, en los que debía reportar emociones, percepciones y expectativas surgidas durante el curso, que se redactaron por medio de unas preguntas guía y fueron examinados a través de una rúbrica. Estos trataron de establecer directamente los factores que los practicantes concibieron como pertinentes, su puesta en práctica en el contexto colombiano y la importancia o las dificultades presentes en el acto de reflexionar frente a sus eventos de clases.

\section{Resultados y discusión}

\subsection{La reflexión como un elemento para el crecimiento profesional de los estudiantes practicantes}

El acto de reflexionar en el aula consiste principalmente en examinar los actos y las técnicas usadas en clase con el propósito de tomar decisiones sobre lo que se debe continuar haciendo y lo que necesita mejorarse. Richards y Lockhart (2007) aseguran que los profesores que son reflexivos acerca de su quehacer en el aula son capaces de indagar sobre su nivel de crecimiento profesional, así como también sobre aquellos aspectos que deben modificarse dentro de la clase. En el siguiente ejemplo tomado de las encuestas, se muestra la respuesta de un practicante que explica el rol determinante que tuvo la reflexión en sus prácticas dirigidas:

WCP3.3: Las reflexiones son un componente esencial en el desarrollo profesional de los profesores y, en nuestro caso, como profesores practicantes, representa una oportunidad de aprender de los errores, una posibilidad de comenzar a ganar experiencias significativas y, de la misma manera, reconocer conductas que de una manera u otra contribuyen o no al desarrollo de nuestra práctica docente.

De acuerdo con lo mencionado en este dato, la reflexión es vista como un elemento fundamental para el crecimiento laboral; además, esta permite descifrar los comporta- 
mientos que a través de las experiencias vividas en clase influencian la praxis de manera positiva 0 negativa. En este aspecto, todos los participantes de las encuestas coincidieron en que la reflexión durante la práctica era importante, usando otros apelativos como esencial y necesaria para el crecimiento profesional. Por lo tanto, la reflexión muestra a los practicantes, de manera oportuna, los aspectos que dentro de sus clases resultaron satisfactorios y específicamente, esta les enseña a ser conscientes de circunstancias que no salieron muy bien en la clase con el propósito de mejorarlas en próximas ocasiones. El siguiente participante describe en el cuestionario virtual la manera en que el reflexionar le ayudó para futuras clases:

WCP3.8: [el reflexionar sobre mis aspectos por mejorar] me ayudaba a buscar ciertas soluciones en próximas ocasiones. Cada vez que una sesión no salía como esperaba sentía un poco de frustración; sin embargo, al tener en cuenta lo que había consignado como aspecto para mejorar y aplicarlo de otra manera en clase, los resultados eran notoriamente mejores.

Conforme a lo dicho en el ejemplo anterior, la reflexión fue una herramienta que contribuyó en el análisis de situaciones que no salieron de la manera esperada y, por ende, permitió tomar medidas para prevenir dichos infortunios en próximas oportunidades. Es decir, cada vez que el practicante escribía una reflexión, esta le permitía ser consciente de los problemas que enfrentaba durante la lección, lo cual lo instaba a encontrar una solución en caso de afrontar situaciones similares en el futuro. De esta manera, él descubría que estos problemas mejoraban al emplear las soluciones planteadas por medio de la reflexión. En cuanto a las demás respuestas, seis de siete estudiantes afirmaron ser constantes al aplicar sus posibles soluciones en reflexiones posteriores, como una manera de brindar mejor calidad a sus alumnos, así como mejorar su quehacer docente durante las prácticas, aprendiendo de los errores.

Sin embargo, un aspecto fundamental para esta investigación fue saber por qué los estudiantes reflexionaban después de cada clase, siendo esta una oportunidad para indagar sobre la verdadera motivación de los estudiantes practicantes para reflexionar sobre sus actos de enseñanza. El siguiente ejemplo compila en una respuesta lo que la mayoría de los practicantes opinaron al respecto:

WCP3.10: Si bien el realizar la reflexión era una tarea de la asignatura Práctica Dirigida y esto representaba un compromiso con la misma, el hecho de hacerla se traducía en una oportunidad de recordar cómo fue mi desempeño y el de las actividades planeadas en clase, así que de una manera u otra esas reflexiones fueron hechas con la finalidad de identificar debilidades y fortalezas de la clase y mejorar en la próxima ocasión. 
Con base en el aporte obtenido por este estudiante, se distinguen dos puntos de vista de lo que representa la reflexión en su quehacer docente. En primer lugar, este constituye un requisito que se tiene en la asignatura Práctica Pedagógica Dirigida, por el cual debía responder como compromiso académico; y en segundo lugar, él afirma que sirve como un instrumento para evocar su propio rendimiento y el de los ejercicios realizados en la clase. En este caso, la mayoría de los entrevistados opinaron lo mismo, expresando el sentido obligatorio de la reflexión, pero a la vez, sumándole mayor importancia a lo que el acto de reflexionar provocaba en su quehacer docente.

\subsection{La estructura de la reflexión de los practicantes participantes}

Después de hacer un análisis sobre las reflexiones escritas por los estudiantes, se pudo determinar el proceso que estas siguieron. Teniendo en cuenta que las preguntas inspiradoras de reflexión propuestas por el curso fueron ¿Qué aspectos positivos fueron encontrados en la clase? ¿Qué aspectos no salieron tan bien? y ¿Qué harías en una próxima oportunidad?, se encontró que 21 de 27 reflexiones describen la situación antes de reflexionar sobre ella. Un ejemplo de ello demuestra cómo antes de clasificar una situación impactante como positiva o negativa, fue necesario hacer una descripción de la actividad que estaban desarrollando:

OFR7: "Los estudiantes tenían que reescribir en la guía a algunos miembros de la familia y unirlos con la respectiva imagen [...] Pudimos notar que los estudiantes lo hicieron muy rápido y fue evidente que ellos entendieron el tema". (Traducción de los autores)

STR9: "Desarrollamos un examen en el cual el estudiante practicante dio la información más relevante sobre cómo resolver el examen. Los estudiantes encontraron atractivo el examen, incluso cuando algunas preguntas eran difíciles de responder [...] Estuve feliz de ver los resultados y de saber que mi esfuerzo no fue una pérdida de tiempo". (Traducción de los autores)

Estos dos ejemplos demuestran la manera en que los participantes iniciaron sus reflexiones, realizando primero una descripción de una situación, lo que les permitió ser conscientes de en qué momentos ocurrió alguna eventualidad. En este aspecto, Farrell (2013) indica que la descripción de incidentes de clase y el posterior análisis de los mismos posibilita al profesor mejorar sus prácticas de enseñanza en el aula. De igual manera, Hussein (2006) señala que la reflexión puede tratarse de simples descripciones de los eventos de clase, como también pueden ser evaluaciones críticas de estos. Así, la descripción de circunstancias ocurridas en el aula puede ser considerada como un tipo de 
reflexión inicial, puesto que aún no ha trascendido al análisis, interpretación y evaluación de los acontecimientos.

Por otro lado, se pudo determinar que 23 de 27 reflexiones analizadas escribían sobre las situaciones que transcurrieron bien y aquellas que necesitaban mejora. Así, se pudo constatar que la mayoría de los practicantes reflexiona sobre estos dos aspectos en el momento de escribir sus reflexiones de clase. Un ejemplo de ello fue encontrado en el siguiente escrito, en el cual la practicante relata las estrategias que en su clase funcionaron bien, así como aquellas que no transcurrieron como se esperaba:

IOR7: “Además, las estrategias de agrupación parecieron haber funcionado muy bien, principalmente porque los estudiantes sintieron alegría y diversión trabajando con un compañero [...] A pesar de que los estudiantes reconocieron la regla del tiempo presente simple en la actividad de re escribir los verbos, ellos obtuvieron malos resultados [...] estos resultados negativos pudieron haber sido porque los estudiantes no tenían un conocimiento completo sobre la estructura gramatical [...]". (Traducción de los autores)

En este sentido, la practicante expresa los buenos resultados que tuvo al implementar cierta estrategia de agrupación, los que pudo evidenciar por medio de los sentimientos expresados por sus alumnos, aunque también indica los lamentables resultados obtenidos en la actividad de escritura. Así, ella analiza la causa de estos últimos, siendo la falta de interiorización de la gramática una de las raíces del problema; sin embargo, en su reflexión no propone posibles soluciones para mejorar dicha situación.

No obstante, algunas reflexiones (17 de 27 reflexiones) describieron la situación que no transcurrió muy bien y presentaron una solución para posteriores clases:

MBR7: "Por otro lado, algunos aspectos no fueron tan buenos. Por ejemplo, aquellos estudiantes que tienen dificultades no quisieron hacer la parte escrita y estuvieron distrayendo a los otros estudiantes [...] La próxima vez yo intentaría asignar parejas de trabajo, un estudiante que sea bien activo y otro que no sea tan activo o que tenga dificultades, para ver cómo funciona”. (Traducción de los autores)

En este caso, la practicante, además de encontrar el núcleo del problema y describir lo que pasó, plantea una solución que le permite evitar esta circunstancia en futuras sesiones. Sobre este punto vale la pena mencionar a Farrell (2013), quien señala que la práctica reflexiva es importante, debido a que los docentes deben compilar datos sólidos de lo que realmente sucede en el aula de clase, para luego reflexionar acerca de esta y posteriormente tomar decisiones basadas en hechos, buscar alternativas y soluciones. Hussein (2006) difiere en este sentido y argumenta que la principal razón de la reflexión en el aula de clase 
no se debe enfocar en buscar soluciones a problemas, sino que debe ser un componente normal del proceso de desarrollo profesional del docente.

Finalmente, la investigación quiso indagar sobre la relevancia que daban los practicantes al incluir teoría referente al área de enseñanza de lenguas dentro de sus reflexiones, lo que indicó que 16 de 27 reflexiones respaldaban con teoría en la cual se pretendía comparar, contrastar y dar validez a algunas técnicas de clase usadas en las prácticas docentes. En el siguiente ejemplo se muestra una manera en que los practicantes incorporaron teoría en sus reflexiones después de clase:

RAMR7: “[...] Yo puse cada juguete dentro, encima, detrás, debajo, etc. de la caja y a los estudiantes les fue pedido que hicieran la oración. De acuerdo con Brown (2001), los profesores necesitan saber los intereses de los estudiantes, usar variedad en las actividades, así como tener sentido del humor para captar la atención de los estudiantes. Esta puede ser considerada una de las actividades más exitosas durante mi práctica, ya que los estudiantes estuvieron muy interesados y con ganas de participar [...]”. (Traducción de los autores)

Según lo que señala la practicante, el haber hecho diferentes actividades usando juguetes pudo ser un factor para que los estudiantes estuvieran interesados en su clase. Así mismo, ella cita un autor que permite constatar la importancia de la variedad de actividades, el sentido del humor y el conocer a los estudiantes como una manera de saber cómo atraer su interés. En este sentido, la docente pretendió dar más validez a las técnicas de enseñanza usadas durante su clase mediante la extrapolación de las ideas de un experto. No obstante, la incorporación de la teoría durante las reflexiones no fue una constante en los estudiantes.

De hecho, en el análisis de los cuestionarios empleados con los practicantes se encontró que seis de siete estudiantes aseguraron haber tenido dificultades en aplicar la teoría sobre la enseñanza de inglés recibida desde la academia en el contexto colombiano. Al respecto, el siguiente resultado resume la relación entre teoría y práctica de acuerdo con los practicantes.

\subsection{La desconexión entre teoría de enseñanza en inglés y la práctica peda- gógica en contextos colombianos}

Cuando se habla de la enseñanza del inglés como lengua extranjera es inevitable acudir a distintos autores que sugieren diferentes enfoques (Richards \& Renandya, 2011) que deben ser fortalecidos durante la enseñanza de la lengua inglesa, como el aprendizaje significativo, la motivación intrínseca y la competencia comunicativa, entre otros. Así mis- 
mo, Thornbury (2005) sugiere que esta enseñanza debe dirigirse a que la lengua emerja en una relación dialógica entre estudiantes y docentes. Sin embargo, los practicantes participantes de esta investigación indican que existe una desconexión entre la teoría estudiada en su carrera y sus experiencias como practicantes en las aulas colombianas, como lo asegura el siguiente ejemplo tomado de un cuestionario virtual realizado a los practicantes cuando se les preguntó si habían encontrado brechas entre las teorías discutidas en su programa y la práctica en las aulas colombianas:

WCP4.2: “[...] Podría decir que en relación con las teorías aprendidas, la mayoría no tiene aplicación en nuestro contexto colombiano. [...] aquí en nuestro país existen problemas socioeconómicos mucho más profundos; en ningún libro que haya leído he podido encontrar qué hacer cuando tu estudiante llega al aula sin haber tomado una gota de agua o comido una miga de pan ¿Cómo motivarlo? Hay cosas que van más a fondo y que para nuestro contexto no existen teorías que nos enseñen en el programa".

Teniendo en consideración la anterior observación, pocas teorías buscan abordar los problemas socioeconómicos como un factor que determine el aprendizaje de una lengua, ya que cuando se habla de motivación se plantea desde un punto de vista diferente de las condiciones económicas que viven a diario los estudiantes en el contexto de las escuelas públicas colombianas. En este sentido, Díaz y Solar (2011) aseguran que las condiciones sociales que los alumnos viven "determinan en cierta medida, las creencias, las actitudes y las aspiraciones que los estudiantes demuestran" (pg.74). En otras palabras, el contexto socioeconómico influye en gran medida sobre la manera en que los estudiantes aprecian el aprendizaje de una lengua, afectando este proceso, en especial cuando las condiciones sociales son adversas.

Un ejemplo tomado del cuestionario virtual realizado a los practicantes resume lo que la mayoría de participantes opina con relación a las brechas encontradas entre teoría y práctica:

WCP4.3: "Si, es notorio cómo en los textos y teorías encontramos unas condiciones casi perfectas para realizar clases, salones con infinidad de materiales o estudiantes con un background [conocimiento previo] más o menos similar. Sin embargo, en las aulas colombianas hay falencia de infinidad de cosas y los estudiantes deben lidiar con una serie de problemas sociales y familiares que de una manera u otra terminan afectando su desempeño en clase. Los estudiantes colombianos tienen diferentes métodos de aprendizaje y en algunos casos es tal vez difícil despertar en ellos autonomía, ya que en la mayoría de casos, nuestra cultura carece de ese factor". 
Lo anterior señala que el estudiante reconoce que las teorías y los textos de los currículos de algunas materias de contenido pedagógico no están relacionados con las condiciones socioculturales, familiares y de infraestructura que se viven en las aulas de clase colombianas, ya que, según él, estos textos académicos se basan en clases de ambientes ideales donde se cuenta con materiales didácticos suficientes y hay estudiantes con muy buenos conocimientos previos. Asimismo, los demás estudiantes practicantes opinaron que buena parte de la teoría no tenía aplicación en el contexto colombiano, pues en ella se proponían condiciones ideales en cuanto a la cantidad de recursos y de estudiantes, así como al manejo de grupo, que difícilmente se parecía a la naturaleza de las aulas colombianas. Y añadieron que estos textos académicos no contextualizan los problemas sociales que pueden ser encontrados en el aula, los cuales son situaciones diarias que deberán enfrentar los docentes en Colombia. De este modo, una mirada más crítica al contexto en el que los estudiantes practicantes deberán encararse en sus prácticas debería ser abordada desde la academia por medio de textos y videos que permitan al practicante ser consciente de la realidad de la educación colombiana.

Sobre este aspecto es importante tener presente la investigación realizada por Akcan (2016), quien reporta que la mayoría de los practicantes participantes en su estudio indicó que les gustaría estar mejor informados acerca de las situaciones reales que encontrarían en su quehacer docente, pues estos consideran que su programa se enfocó más en la teoría que en la práctica, y que los docentes del programa deberían exponerlos a las condiciones reales del contexto educativo con el propósito de prepararlos mejor. De igual manera, este autor (2016) señala que los programas de educación deben conectar la teoría general con la práctica específica, para que los futuros docentes puedan estar mejor preparados para lidiar con la realidad de sus aulas de clase.

\section{Conclusiones}

A través de una de las preguntas que guiaron esta investigación (¿Cuál es la percepción de los practicantes acerca de la reflexión como parte de su crecimiento profesional como docentes de inglés?), se pudieron descubrir los pensamientos que algunos de los participantes expresaron al respecto. Los practicantes manifestaron la importancia de la reflexión en su crecimiento profesional como docentes de inglés para ser conscientes de sus fortalezas y aspectos por mejorar, así como para reconocer conductas que pueden afectar la clase y encontrar soluciones a estas. Teniendo en cuenta que la reflexión después de clase era un ejercicio obligatorio y por el cual obtendrían una nota, algunos docentes noveles opinaron que una de sus motivaciones para reflexionar era el obtener una buena nota. Sin embargo, el carácter formativo y la capacidad para hacerlos conscientes de sus 
errores y de las soluciones que se podrían implementar en estos casos, fueron sus mayores razones para reflexionar después de clase.

Por otro lado, con la pregunta ¿qué conjunto de acciones describen o incorporan los practicantes en sus reflexiones después de clase? se pudo determinar que, en su gran mayoría, los participantes describen las situaciones antes de reflexionar sobre ellas, señalan las acciones positivas y por mejorar encontradas en la lección, y solo algunos proponen soluciones para frenar comportamientos o acciones en próximas oportunidades, e incorporan aportes teóricos para fundamentar o contrastar sus técnicas de enseñanza.

Por otra parte, comparando las preguntas inspiradoras de reflexión usadas en el curso de práctica dirigida (¿Qué salió bien durante la sesión? ¿Qué lo evidencia? ¿Qué aspecto debe mejorarse? y ¿Qué podría hacerse en próximas sesiones?) con los niveles de reflexión planteados por Hatton y Smith (1995), se pudo determinar que estas preguntas solo permiten alcanzar en los practicantes el primer nivel de reflexión (reflexión técnica), en el que el objetivo principal es inspirar a que los estudiantes descubran problemas presentados durante la clase para así buscar soluciones. De este modo, el practicante usa sus habilidades y conocimientos técnicos en el área para corregir y controlar una situación. Debido a esto, los estudiantes del curso tendrían pocas oportunidades para trascender al siguiente nivel (reflexión práctica), puesto que este tipo de preguntas no permite indagar el porqué de las situaciones y acciones presentadas en la clase, ni explicar o confrontar sus propias creencias o conocimientos adquiridos en la carrera con la realidad vivida en el aula. Con base en esto, se concluye que los estudiantes practicantes del curso no llegan a niveles superiores por falta de un proceso que los conduzca a entender qué es la reflexión, cómo hacerla y a qué niveles se espera que lleguen. Así mismo, el profesor director de curso debe plantear distintas herramientas que permitan que el practicante elija el modo en que desea reflexionar y sobre qué enfoque le gustaría trabajar.

Por último, un enfoque más realista de cómo es enseñar inglés en el contexto educativo colombiano debe ser abordado desde la academia, de modo que el docente en formación se aproxime de manera más lógica por medio de la teoría a la realidad a la cual se enfrentará una vez inicie sus prácticas docentes. Así, entonces, el practicante estará lo suficientemente preparado y tendrá mejores herramientas para afrontar la realidad del sector educativo colombiano. 


\section{Referencias}

Akcan, S. (2016). Novice Non-Native English Teachers' Reflections on their Teacher Education Programmes and their First Years of Teaching. Profile Issues in Teachers' Professional Development, 18(1), 55-70. https://doi.org/10.15446/profile.v18n1.48608

Cross, P., \& Angelo, T. (1988). Classroom Assessment Techniques. A Handbook for Faculty (1st ed., Vol. 1). Ann Arbor, Michigan: Jossey-Bass.

Delors, J. (1999). Los cuatro pilares de la educación. La educación encierra un tesoro. París: El Correo de la Unesco.

Dewey, J. (1933). How we think. The Problem of Training Thought. Boston, D. C.: Heath \& Co.

Díaz, C., \& Solar, M.I. (2011). La revelación de las creencias lingüístico-pedagógicas a partir del discurso del profesor de inglés universitario. RLA. Revista de Lingüística Teórica y Aplicada, 49(2), 74. http://dx.doi.org/10.4067/S0718-48832011000200004

Domingo, A., \& Gómez, V. (2015). La práctica reflexiva. Bases, modelos e instrumentos. Revista Complutense de Educación, 26(2), 485-494. Recuperado de https://revistas.ucm.es/index. php/RCED/issue/view/2699/showToc

Faez, F., \& Valeo, A. (2012). TESOL Teacher Education: Novice Teachers' Perceptions of Their Preparedness and Efficacy in the Classroom. TESOL Quarterly, 46(3), 450-471.

Farrel, T. (2013). Evidence-Based Reflective Practice. Retrieved from https://earlyreflections1.files. wordpress.com/2016/06/etas-j-spring-2013_ss-evidence-based-rp.pdf

Fekede, T. \& Gemechis F. (2009). Practicum Experience in Teacher Education. Ethiopain Journal of Education and Sciences of Jimma University, 5(1), 107-116.

Fraenkel, J., Wallen, N., \& Hyun, H. (2012). How to Design and Evaluate Research in Education (8 ${ }^{\text {th }}$ ed.). San Francisco, CA: San Francisco State University, McGraw-Hill.

García, D., \& García, J. (2014). Educación bilingüe y pluralidad: reflexiones en torno de la interculturalidad crítica. Cuadernos de Lingüística Hispánica, (23), 49-65.

Ghaye, A., \& Ghaye, K. (1998). Teaching and Learning through Critical Reflective Practice. London: David Fulton.

Greene, M. (1986). Reflection and Passion in Teaching. Journal of Curriculum and Supervision, $2(1), 68-81$.

Hatton, N. \&Smith,D. (1995). Reflection in Teacher Education: Towards Definition and Implementation. Teaching and Teacher Education, 11(1), 33-39.

Hussein, J. W. (2006). Which one is better: Saying Student Teachers don't reflect or systematically unlocking their Reflective Potentials: A Positive Experience from a Poor Teacher Education Faculty in Ethiopia. Australian Journal of Teacher Education, 31(2). 
Killion, J., \& Todnem, G. (1991). A Process of Personal Theory Building. Educational Leadership, 48(6), 14-17.

Lodico, M.G., Spaulding, D.T., \& Voegtle, K. H. (2006). Methods in Educational Research: From Theory to Practice ( $3^{\text {rd }}$ ed.). Vol. 1, (pp. 237- 697). USA: Jossey-Bass.

Lortie, D. (1975). Schoolteacher: A Sociological Study. Chicago: The University of Chicago Press.

Madrid, D. (2004). La formación inicial del profesorado de lengua extranjera. Profesorado, Revista de Currículumy Formación del Profesorado, 8(1). Recuperado de https://www.ugr.es/ recfpro/ rev81ART7.pdf.

Ministerio de Educación Nacional. (2016). Resolución 02041 de 2016. Bogotá: MEN.

Munby, H., \& Russell, T. (1990). Metaphor in the Study of Teachers' Professional Knowledge. Theory into Practice, 29(2), 116-121.

Páez, V. (2001). El profesor de idiomas: sus cualidades y competencias. Comunicación, 11(3).

Peters, R. (1966). Ethics and Education. London: Allen \& Unvin.

Pitton, D.E. (2006). Defining the Mentoring Relationship. In Mentoring Novice Teachers (pp. 1-18). CA, USA: Corwin Press.

Richards, J. C., \& Lockhart, C. (2007). Reflective Teaching in Second Language Classrooms. New York: Cambridge University Press.

Richards, J. C., \& Renandya, W. A. (2011). Methodology in language teaching: an anthology of current practice. New York: Cambridge University Press.

Shön, D. (1984). The Reflective Practitioner: How Professionals Think in Action. New York: Basic Books.

Schön, D. (1987). Educating the Reflective Practitioner. San Francisco: Jossey-Bass.

Thornbury, S. (2005). Dogme: Dancing in the Dark? Humanising Language Teaching, 7, 2

Ur, P. (1999). A Course in Language Teaching of Theory (17th ed., Vol. 1). Cambridge: Cambridge University Press.

Veenman, S., De Laat, H. \& Staring, C. (1998). Coaching Beginning Teachers, Ljubljana. Recuperado de http://files.eric.ed.gov/fulltext/ED426967.pdf

Zeichner, K. \& Liston, D. (1996). Historical Roots of Reflective Teaching. Reflective Teaching. An Introduction. New Jersey, USA: Lawrence Erlbaum Associates. 